\title{
Output Feedback Stabilization of Linear Systems with Actuator Saturation
}

\author{
Fen $\mathrm{Wu}^{1}$, Zongli Lin ${ }^{2}$ and Qian Zheng ${ }^{3}$
}

\begin{abstract}
The paper presents a method for designing output feedback laws that stabilize a linear system subject to actuator saturation with a large domain of attraction. This method applies to general linear systems including strictly unstable ones, and is presented in both continuous-time and discrete-time setting. A nonlinear output feedback controller is first expressed in the form of a quasi-LPV system. Conditions under which the closed-loop system is locally asymptotically stable are then established in terms of the coefficient matrices of the controller. The design of the controller (coefficient matrices) that achieves a large domain of attraction is then formulated and solved as an optimization problem with LMI constraints.
\end{abstract}

\section{INTRODUCTION}

In this paper, we consider the basic problem of stabilizing a linear system subject to input saturation by use of output feedback. The stabilization of a linear system subject to actuator saturation has been widely reported on in the literature. The works on this topic can be divided into two categories, those that deal with open-loop systems that are not exponentially unstable (or simply called semi-stable) and those that are exponentially unstable. The stabilization of semi-stable systems is now well-understood. Various global and semi-global stabilizing feedback laws, both of state feedback type and output feedback type, have been reported (see, e.g., [7], [8], [10], [11], [12]).

The stabilization of exponentially unstable systems subject to actuator saturation involves subtler issues and is more difficult to deal with. The fundamental difference between a semi-stable system under actuator saturation and an exponentially unstable linear system under actuator saturation lies in their respective null controllable region, the set of all states that can be driven to the origin by a bounded control (provided by the saturating actuator). In particular, the null controllable region of a semi-stable linear controllable system is the whole state space, while the null controllable region of an exponentially unstable linear controllable system is an open set in the state space (see, e.g., [3]). Therefore, in controlling exponentially unstable linear systems under actuator saturation, one can only expect local stabilization. The natural objective is then to achieve a

1. Department of Mechanical and Aerospace Engineering, North Carolina State University, Raleigh, NC 27695. Email: fwu@eos.ncsu.edu. Work supported in part by NSF Grant CMS-0324397.

2. Charles L. Brown Department of Electrical and Computer Engineering, University of Virginia, Charlottesville, VA 22904. Email: z15y@ virginia.edu. Work supported in part by NSF Grant CMS-0324329.

3. Department of Mechanical and Aerospace Engineering, North Carolina State University, Raleigh, NC 27695. Email: qzheng@unity.ncsu.edu. Work supported in part by NSF Grant CMS-0324397. domain of attraction that is as large as possible and, ideally, as large as the null controllable region of the system. It is however known that a domain of attraction as large as the null controllable region in general cannot be achieved with linear feedback [4]. As a result, many methods exist in the literature for the synthesis of linear state feedback laws that result in large domains of attraction (see, e.g., [2], [6], [9]). More recently, an LMI based approach for the design of stabilizing state feedback law was proposed in [5]. This method was shown to result in larger domains of attraction than other existing methods. The key novelties of this method include putting the saturating linear feedback law on the convex hull involving an auxiliary feedback gain matrix and the establishment of set invariance conditions that are equivalent to linear matrix inequalities.

The objective of this paper is to develop a method for the synthesis of stabilizing output feedback laws. The feedback law that we will arrive at will be nonlinear in nature. By utilizing convex hull expression of saturating linear feedback law [5], we will first parameterize the proposed nonlinear output feedback law in the form of a quasi-LPV system. Conditions under which the closed-loop system is locally asymptotically stable at the origin with a Lyapunov level set included in the domain of attraction are then established in terms of the coefficient matrices of the controller in the quasi-LPV form. The design of these controller coefficient matrices is then formulated and solved as an LMI optimization problem.

The notation used in this paper is fairly standard. $\mathbf{R}$ stands for the set of real numbers and $\mathbf{R}_{+}$for the nonnegative real numbers. $\mathbf{R}^{m \times n}$ is the set of real $m \times n$ matrices. The transpose of a real matrix $M$ is denoted by $M^{\mathrm{T}}$. We use $\mathbf{S}^{n \times n}$ to denote real, symmetric $n \times n$ matrices, and $\mathbf{S}_{+}^{n \times n}$ for positive definite matrices. If $M \in \mathbf{S}^{n \times n}$, then $M>0(M \geq 0)$ indicates that $M$ is a positive definite (positive semi-definite) matrix and $M<0(M \leq 0)$ denotes a negative definite (negative semi-definite) matrix. A block diagonal matrix with matrices $X_{1}, X_{2}, \cdots, X_{p}$ on its diagonal is denoted by $\operatorname{diag}\left\{X_{1}, X_{2}, \cdots, X_{p}\right\}$. For two integers $k_{1}, k_{2}, k_{1}<k_{2}$, we denote $\left[k_{1}, k_{2}\right]=\left\{k_{1}, k_{1}+1, \cdots, k_{2}\right\}$.

The remainder of this paper is organized as follows. The main results on the synthesis of output feedback laws are presented in Section II, where both the continuous-time and discrete-time systems are considered. Section III contains a numerical example to illustrate our design procedure and the effectiveness of the resulting controller. Section IV draws the conclusion to the paper. 


\section{Synthesis of Output Feedback Stabilizing LAWS}

\section{A. Continuous-Time Systems}

Consider a continuous-time linear time-invariant system subject to actuator saturation

$$
\left\{\begin{aligned}
\dot{x}_{\mathrm{p}} & =A_{\mathrm{p}} x_{\mathrm{p}}+B_{\mathrm{p}} \sigma(u), \\
y & =C_{\mathrm{p}} x_{\mathrm{p}},
\end{aligned}\right.
$$

where $x_{\mathrm{p}} \in \mathbf{R}^{n}$ is the state, $y \in \mathbf{R}^{p}$ the measurement, $u \in \mathbf{R}^{m}$ the control input, and the matrix triple $\left(A_{\mathrm{p}}, B_{\mathrm{p}}, C_{\mathrm{p}}\right)$ are stabilizable and detectable. Also, the function $\sigma: \mathbf{R}^{m} \rightarrow$ $\mathbf{R}^{m}$ is a vector valued standard saturation function, i.e.,

$$
\sigma(u)=\left[\begin{array}{llll}
\sigma\left(u_{1}\right) & \sigma\left(u_{2}\right) & \cdots & \sigma\left(u_{m}\right)
\end{array}\right]^{\mathrm{T}},
$$

with

$$
\sigma\left(u_{i}\right)=\operatorname{sgn}\left(u_{i}\right) \min \left\{1,\left|u_{i}\right|\right\} .
$$

Here, we have slightly abused the notation by using $\sigma$ to denote both the scalar valued and vector valued saturation function. Also, we note that it is without loss of generality to assume a unity saturation level, as level of saturation can always be scaled to unity by scaling $B_{\mathrm{p}}$ and $u$.

We will consider a dynamic output feedback law of the form,

$$
\left\{\begin{aligned}
\dot{x}_{\mathrm{c}} & =A_{\mathrm{c}}\left(x_{\mathrm{c}}, y\right) x_{\mathrm{c}}+B_{\mathrm{c}}\left(x_{\mathrm{c}}, y\right) y, \quad x_{\mathrm{c}} \in \mathbf{R}^{n_{\mathrm{c}}}, \\
u & =C_{\mathrm{c}} x_{\mathrm{c}}+D_{\mathrm{c}} y,
\end{aligned}\right.
$$

where the controller dimension $n_{\mathrm{c}}=n, C_{\mathrm{c}}$ and $D_{\mathrm{c}}$ are constant matrices of appropriate dimensions.

Our design objective is to construct a dynamic output feedback law of the form (2) that asymptotically stabilizes the plant (1) at the origin with a domain of attraction as large as possible. This objective is achieved as follows. We will first parameterize the controller in a quasi-LPV form and then establish conditions on the coefficient matrices of the parameterized controller under which the closedloop system is asymptotically stable at the origin with an ellipsoid of the form

$$
\Omega(X, \beta)=\left\{x \in \mathbf{R}^{n+n_{\mathrm{c}}}: x^{\mathrm{T}} X x \leq \beta\right\}, \quad X>0, \beta>0
$$

contained in the domain of attraction. The determination of the controller coefficient matrices is then formulated and solved as an LMI optimization problem with the objective of maximizing the ellipsoid $\Omega(X, \beta)$.

To this end, we will need to use a tool from [3] for expressing the saturated linear feedback $\sigma\left(C_{\mathrm{c}} x_{\mathrm{c}}+D_{\mathrm{c}} y\right)$ on a convex hull. For $H_{C} \in \mathbf{R}^{m \times n}$ and $H_{D} \in \mathbf{R}^{m \times p}$, define

$$
\begin{gathered}
\mathcal{L}\left(H_{C}, H_{D}\right)=\left\{\left(x_{\mathrm{c}}, y\right) \in \mathbf{R}^{n_{\mathrm{c}}+p}:\left|H_{C_{i}} x_{\mathrm{c}}+H_{D_{i}} y\right| \leq 1,\right. \\
i \in[1, m]\},
\end{gathered}
$$

where $H_{C i}$ and $H_{D i}$ represent the $i$ th row of matrices $H_{C}$ and $H_{D}$ respectively. We note that $\mathcal{L}\left(H_{C}, H_{D}\right)$ represents the region in $\mathbf{R}^{n_{\mathrm{c}}+p}$ where the auxiliary feedback $H_{C} x_{\mathrm{c}}+$ $H_{D} y$ does not saturate.
Also, let $\mathcal{V}$ be the set of $m \times m$ diagonal matrices whose diagonal elements are either 1 or 0 . There are $2^{m}$ elements in $\mathcal{V}$. Suppose these elements of $\mathcal{V}$ are labeled as $E_{j}, j \in$ $\left[0,2^{m}-1\right]$, where for $j=z_{1} 2^{m-1}+z_{2} 2^{m-2}+\cdots+z_{m}$ with $z_{i} \in\{0,1\}$, the diagonal elements of $E_{j}$ are $\left\{1-z_{1}, 1-\right.$ $\left.z_{2}, \cdots, 1-z_{m}\right\}$. Denote $E_{j}^{-}=I-E_{j}$. Clearly, $E_{j}^{-} \in \mathcal{V}$ if $E_{j} \in \mathcal{V}$.

The following lemma is adopted from [3].

Lemma 1: For $\left(x_{\mathrm{c}}, y\right) \in \mathcal{L}\left(H_{C}, H_{D}\right)$,

$$
\begin{aligned}
\sigma\left(C_{\mathrm{c}} x_{\mathrm{c}}+D_{\mathrm{c}} y\right) & \in \operatorname{co}\left\{E_{j}\left(C_{\mathrm{c}} x_{\mathrm{c}}+D_{\mathrm{c}} y\right)\right. \\
& \left.+E_{j}^{-}\left(H_{C} x_{\mathrm{c}}+H_{D} y\right), j \in\left[0,2^{m}-1\right]\right\},
\end{aligned}
$$

where co stands for the convex hull.

For single saturation case (i.e., $m=1$ ), the convex covering in the above lemma is non-conservative as shown in [3]. In general, Lemma 1 provides more stringent convex covering of saturation nonlinearities than other existing approaches [2], [9]. As a result, the stability conditions derived from this lemma will be less conservative.

By Lemma 1, the saturated linear feedback, with $\left(x_{\mathrm{c}}, y\right) \in$ $\mathcal{L}\left(H_{C}, H_{D}\right)$, can be expressed as

$$
\begin{aligned}
\sigma\left(C_{\mathrm{c}} x_{\mathrm{c}}+D_{\mathrm{c}} y\right) & \sum_{j=0}^{2^{m}-1} \eta_{j}\left[E_{j}\left(C_{\mathrm{c}} x_{\mathrm{c}}+D_{\mathrm{c}} y\right)\right. \\
& \left.+E_{j}^{-}\left(H_{C} x_{\mathrm{c}}+H_{D} y\right)\right],
\end{aligned}
$$

for some scalars $0 \leq \eta_{j} \leq 1, j \in\left[0,2^{m}-1\right]$, such that $\sum_{i=0}^{2^{m}-1} \eta_{j}=1$. We note that the scalars $\eta_{j}$ 's are functions of $x_{\mathrm{c}}$ and $y$ and their values are available in real-time. These scalars in a way reflect the severity of control saturation. In general, there are multiple choices of $\eta_{j}$ 's satisfying the same constraint, leading to non-unique representation of (3). For example, when $m=2$, the values of $\eta_{j}$ 's can be determined as follows. For $i=1,2$, let

$\lambda_{i}=\left\{\begin{array}{l}1 \quad \text { if } C_{\mathrm{c} i} x_{\mathrm{c}}+D_{\mathrm{c} i} y=H_{C_{i}} x_{\mathrm{c}}+H_{D_{i}} y, \\ \frac{\sigma\left(u_{i}\right)-\left(H_{C i} x_{\mathrm{c}}+H_{D_{i}} y\right)}{\left(C_{\mathrm{c} i}-H_{D i}\right) x_{\mathrm{c}}+\left(D_{\mathrm{c} i}-H_{D i}\right) y} \text { otherwise. }\end{array}\right.$

Also define

$$
\left\{\begin{array}{l}
\eta_{0}=\lambda_{1} \lambda_{2}, \\
\eta_{1}=\lambda_{1}\left(1-\lambda_{2}\right), \\
\eta_{2}=\left(1-\lambda_{1}\right) \lambda_{2}, \\
\eta_{3}=\left(1-\lambda_{1}\right)\left(1-\lambda_{2}\right) .
\end{array}\right.
$$

Clearly, each $\eta_{j} \in[0,1]$ and $\sum_{j=0}^{3} \eta_{j}=1$.

We note that $\eta_{j}\left(x_{c}, y\right)$ 's are locally Lipschitz in their arguments. For higher order systems with inputs more than two, $\eta_{j}$ 's can be defined in a similar way.

We will use the functions $\eta_{j}\left(x_{\mathrm{c}}, y\right)$ 's to parameterize the output feedback control (2) into the following quasi-LPV system,

$$
\left\{\begin{aligned}
\dot{x}_{\mathrm{c}} & =\left(\sum_{j=0}^{2^{m}-1} \eta_{j} A_{\mathrm{c} j}\right) x_{\mathrm{c}}+\left(\sum_{j=0}^{2^{m}-1} \eta_{j} B_{\mathrm{c} j}\right) y \\
u & =C_{\mathrm{c}} x_{\mathrm{c}}+D_{\mathrm{c}} y
\end{aligned}\right.
$$


where the coefficient matrices $A_{\mathrm{c} j}$ 's, $B_{\mathrm{c} j}$ 's, $C_{\mathrm{c}}$ and $D_{\mathrm{c}}$ are to be designed. Since $\eta_{j}$ 's are time varying parameters which will be used to schedule the controller gain, the resulting controller is called a gain scheduling controller.

We can also write the plant (1) in a quasi-LPV form as follows,

$$
\left\{\begin{array}{ccc}
\dot{x}_{\mathrm{p}}= & A_{\mathrm{p}} x_{\mathrm{p}}+B_{\mathrm{p}} \sum_{j=0}^{2^{m}-1} \eta_{j}\left[E_{j}\left(C_{\mathrm{c}} x_{\mathrm{c}}+D_{\mathrm{c}} y\right)\right. \\
& \left.\quad+E_{j}^{-}\left(H_{C} x_{\mathrm{c}}+H_{D} y\right)\right] \\
y= & C_{\mathrm{p}} x_{\mathrm{p}} .
\end{array}\right.
$$

Motivated by the quasi-LPV structure of both the plant and the controller, we consider the following auxiliary LPV system, of which the closed-loop system comprising of (6) and (5) is a special case, if $\Omega(X, \beta) \subset \mathcal{L}\left(H_{D} C_{\mathrm{p}}, H_{C}\right)$ is an invariant set,

$$
\dot{x}=A(\eta) x, \quad \eta \in \Gamma,
$$

where

$$
\begin{array}{r}
\eta=\left[\eta_{0}, \eta_{1}, \cdots, \eta_{2^{m}-1}\right], \quad x=\left[\begin{array}{l}
x_{\mathrm{p}} \\
x_{\mathrm{c}}
\end{array}\right], \\
\Gamma=\left\{\eta \in \mathbf{R}^{2^{m}}: \sum_{j=0}^{2^{m}-1} \eta_{j}=1,0 \leq \eta_{j} \leq 1,\right. \\
\left.j \in\left[0,2^{m}-1\right]\right\},
\end{array}
$$

and

$$
\begin{aligned}
A(\eta) & =\left[\begin{array}{cc}
A_{\mathrm{p}} & 0 \\
0 & 0
\end{array}\right]+\left[\begin{array}{cc}
0 & B_{\mathrm{p}} \\
I & 0
\end{array}\right] \\
\times & \left(\sum_{j=0}^{2^{m}-1} \eta_{j}\left[\begin{array}{cc}
A_{\mathrm{c} j} & B_{\mathrm{c} j} \\
E_{j} C_{\mathrm{c}}+E_{j}^{-} H_{C} & E_{j} D_{\mathrm{c}}+E_{j}^{-} H_{D}
\end{array}\right]\right)\left[\begin{array}{cc}
0 & I \\
C_{\mathrm{p}} & 0
\end{array}\right] \\
& :=\sum_{i=0}^{2^{m}-1} \eta_{j} A_{j} .
\end{aligned}
$$

The following theorem establishes conditions on the controller coefficient matrices under which the LPV system (8) is asymptotically stable with a quadratic Lyapunov function.

Theorem 1: Consider the closed-loop system (8). If there exist positive definite matrices $R, S \in \mathbf{S}_{+}^{n \times n}$ and matrices $\left(\bar{A}_{\mathrm{c} j}, \bar{B}_{\mathrm{c} j}\right) \in \mathbf{R}^{n \times n} \times \mathbf{R}^{n \times p}, j \in\left[0,2^{m}-1\right],\left(\bar{C}_{\mathrm{c}}, \bar{D}_{\mathrm{c}}\right) \in$ $\mathbf{R}^{m \times n} \times \mathbf{R}^{m \times p}$ and $\left(\bar{H}_{C}, \bar{H}_{D}\right) \in \mathbf{R}^{m \times n} \times \mathbf{R}^{m \times p}$ such that

$$
\begin{aligned}
& {\left[\begin{array}{cc}
\left\{\begin{array}{c}
A_{\mathrm{p}} R+B_{\mathrm{p}} \bar{K}_{\mathrm{c} j} \\
+R A_{\mathrm{p}}^{\mathrm{T}}+\bar{K}_{\mathrm{c} j}^{\mathrm{T}} B_{\mathrm{p}}^{\mathrm{T}}
\end{array}\right\} & \star \\
\bar{A}_{\mathrm{c} j}+A_{\mathrm{p}}^{\mathrm{T}}+C_{\mathrm{p}}^{T} \bar{L}_{\mathrm{c} j}^{\mathrm{T}} B_{\mathrm{p}}^{\mathrm{T}} & \left.\begin{array}{c}
A_{\mathrm{p}}^{\mathrm{T}} S+C_{\mathrm{p}}^{\mathrm{T}} \bar{B}_{\mathrm{c} j}^{\mathrm{T}} \\
+S A_{\mathrm{p}}+\bar{B}_{\mathrm{c} j} C_{\mathrm{p}}
\end{array}\right\} \\
j \in\left[0,2^{m}-1\right],
\end{array}\right.} \\
& {\left[\begin{array}{cc}
R & I \\
I & S
\end{array}\right]>0,}
\end{aligned}
$$

where $\bar{K}_{\mathrm{c} j}=E_{j} \bar{C}_{\mathrm{c}}+E_{j}^{-} \bar{H}_{C}$ and $\bar{L}_{\mathrm{c} j}=E_{j} \bar{D}_{\mathrm{c}}+E_{j}^{-} \bar{H}_{D}$, then, with the following controller coefficient matrices for

$$
\begin{aligned}
j \in\left[0,2^{m}-1\right] & \left.\begin{array}{cc}
A_{\mathrm{c} j} & B_{\mathrm{c} j} \\
C_{\mathrm{c}} & D_{\mathrm{c}} \\
H_{C} & H_{D}
\end{array}\right]= \\
& {\left[\begin{array}{ccc}
N & S B_{\mathrm{p}} E_{j} & S B_{\mathrm{p}} E_{j}^{-} \\
0 & I_{m} & 0 \\
0 & 0 & I_{m}
\end{array}\right]^{-1}\left\{\left[\begin{array}{cc}
\bar{A}_{\mathrm{c} j} & \bar{B}_{\mathrm{c} j} \\
\bar{C}_{\mathrm{c}} & \bar{D}_{\mathrm{c}} \\
\bar{H}_{C} & \bar{H}_{D}
\end{array}\right]\right.} \\
& \left.-\left[\begin{array}{cc}
S A_{\mathrm{p}} R & 0 \\
0 & 0 \\
0 & 0
\end{array}\right]\right\}\left[\begin{array}{cc}
M^{\mathrm{T}} & 0 \\
C_{\mathrm{p}} R & I_{p}
\end{array}\right]^{-1}, \quad(11)
\end{aligned}
$$

where $M, N \in \mathbf{R}^{n \times n}$ are such that $M N^{\mathrm{T}}=I_{n}-R S$, the closed-loop system (8) is asymptotically stable. Moreover,

$$
A^{\mathrm{T}}(\eta) X+X A(\eta)<0, \quad \forall \eta \in \Gamma,
$$

where $X>0$ is given by

$$
X=\left[\begin{array}{cc}
I & S \\
0 & N^{\mathrm{T}}
\end{array}\right]\left[\begin{array}{cc}
R & I \\
M^{\mathrm{T}} & 0
\end{array}\right]^{-1} .
$$

Proof: We first show that $X$ as defined in (13) is indeed positive definite. To this end, let

$$
Z_{1}=\left[\begin{array}{cc}
R & I \\
M^{\mathrm{T}} & 0
\end{array}\right], \quad Z_{2}=\left[\begin{array}{cc}
I & S \\
0 & N^{\mathrm{T}}
\end{array}\right],
$$

which are both nonsingular, then $X Z_{1}=Z_{2}$ and

$$
Z_{1}^{\mathrm{T}} X Z_{1}=Z_{2}^{\mathrm{T}} Z_{1}=\left[\begin{array}{cc}
R & I \\
I & S
\end{array}\right]>0,
$$

which implies that

$$
X=\left(Z_{1}^{-1}\right)^{\mathrm{T}}\left[\begin{array}{cc}
R & I \\
I & S
\end{array}\right] Z_{1}^{-1}>0 .
$$

We next show that $X$ satisfies the inequality (12). Using the fact that $X Z_{1}=Z_{2}$ again, we have that, for each $j \in$ $\left[0,2^{m}-1\right]$,

$$
\begin{aligned}
& Z_{1}^{\mathrm{T}}\left(X A_{j}\right) Z_{1}=Z_{2}^{\mathrm{T}} A_{j} Z_{1} \\
& =\left[\begin{array}{cc}
A_{\mathrm{p}} R & A_{\mathrm{p}} \\
0 & S A_{\mathrm{p}}
\end{array}\right]+\left[\begin{array}{cc}
0 & B_{\mathrm{p}} \\
I & 0
\end{array}\right]\left[\begin{array}{cc}
\bar{A}_{\mathrm{c} j} & \bar{B}_{\mathrm{c} j} \\
\bar{K}_{\mathrm{c} j} & \bar{L}_{\mathrm{c} j}
\end{array}\right]\left[\begin{array}{cc}
I & 0 \\
0 & C_{\mathrm{p}}
\end{array}\right],
\end{aligned}
$$

where the transformed controller data $\left(\bar{A}_{\mathrm{c} j}, \bar{B}_{\mathrm{c} j}, \bar{C}_{\mathrm{c}}, \bar{D}_{\mathrm{c}}\right.$, $\left.\bar{H}_{C}, \bar{H}_{D}\right)$ relates to the original controller data $\left(A_{\mathrm{c} j}, B_{\mathrm{c} j}, C_{\mathrm{c}}, D_{\mathrm{c}}, H_{C}, H_{D}\right)$ by

$$
\begin{aligned}
{\left[\begin{array}{cc}
\bar{A}_{\mathrm{c} j} & \bar{B}_{\mathrm{c} j} \\
\bar{C}_{\mathrm{c}} & \bar{D}_{\mathrm{c}} \\
\bar{H}_{C} & \bar{H}_{D}
\end{array}\right]=} & {\left[\begin{array}{cc}
S A_{\mathrm{p}} R & 0 \\
0 & 0 \\
0 & 0
\end{array}\right]+\left[\begin{array}{ccc}
N & S B_{\mathrm{p}} E_{j} & S B_{\mathrm{p}} E_{j}^{-} \\
0 & I & 0 \\
0 & 0 & I
\end{array}\right] } \\
& \times\left[\begin{array}{cc}
A_{\mathrm{c} j} & B_{\mathrm{c} j} \\
C_{\mathrm{c}} & D_{\mathrm{c}} \\
H_{C} & H_{D}
\end{array}\right]\left[\begin{array}{cc}
M^{\mathrm{T}} & 0 \\
C_{\mathrm{p}} R & I
\end{array}\right] .
\end{aligned}
$$

We note here that both matrices

$$
\left[\begin{array}{ccc}
N & S B_{\mathrm{p}} E_{j} & S B_{\mathrm{p}} E_{j}^{-} \\
0 & I & 0 \\
0 & 0 & I
\end{array}\right], \quad\left[\begin{array}{cc}
M^{\mathrm{T}} & 0 \\
C_{\mathrm{p}} R & I
\end{array}\right]
$$

are nonsingular. 
It then follows from (14) and (9) that, for each $j \in$ $\left[0,2^{m}-1\right]$,

$$
\begin{aligned}
& Z_{1}^{\mathrm{T}}\left(A_{j}^{\mathrm{T}} X+X A_{j}\right) Z_{1} \\
& =\left[\begin{array}{c}
\left\{\begin{array}{c}
A_{\mathrm{p}} R+B_{\mathrm{p}} \bar{K}_{\mathrm{c} j} \\
+R A_{\mathrm{p}}^{\mathrm{T}}+\bar{K}_{\mathrm{c} j}^{\mathrm{T}} B_{\mathrm{p}}^{\mathrm{T}}
\end{array}\right\} \\
\bar{A}_{\mathrm{c} j}+A_{\mathrm{p}}^{\mathrm{T}}+C_{\mathrm{p}}^{\mathrm{T}} \bar{L}_{\mathrm{c} j}^{\mathrm{T}} B_{\mathrm{p}}^{\mathrm{T}}
\end{array} \quad\left\{\begin{array}{c}
A_{\mathrm{p}}^{\mathrm{T}} S+C_{\mathrm{p}}^{\mathrm{T}} \bar{B}_{\mathrm{c} j}^{\mathrm{T}} \\
+S A_{\mathrm{p}}+\bar{B}_{\mathrm{c} j} C_{\mathrm{p}}
\end{array}\right\}\right]<0,
\end{aligned}
$$

which implies that

$$
A_{j}^{\mathrm{T}} X+X A_{j}<0
$$

Taking linear combination of (16) over $j \in\left[0,2^{m}-1\right]$ with coefficients $\eta_{j}$ yields (12), i.e.,

$$
A^{\mathrm{T}}(\eta) X+X A(\eta)<0, \quad \forall \eta \in \Gamma .
$$

Finally, to show the asymptotic stability of the closedloop system (8), we consider the quadratic Lyapunov function $V(x)=x^{\mathrm{T}} X x$. Taking derivative of $V(x)$ along the trajectories of (8) yields

$$
\dot{V}=x^{\mathrm{T}}\left[A^{\mathrm{T}}(\eta) X+X A(\eta)\right] x<0,
$$

for all $x \in \mathbf{R}^{n+n_{\mathrm{c}}} \backslash\{0\}$ and $\eta \in \Gamma$, which implies asymptotic stability of the closed-loop system.

Recalling that the quasi-LPV representation of the plant (6) and controller (5) was obtained under the assumption that $\left(x_{\mathrm{c}}, y\right) \in \mathcal{L}\left(H_{C}, H_{D}\right)$, thus we have the following result on output stabilization of the plant (1) using a full-order controller (2).

Theorem 2: Consider the plant (1). If there exist positive definite matrices $R, S \in \mathbf{S}_{+}^{n \times n}$ and matrices $\left(\bar{A}_{\mathrm{c} j}, \bar{B}_{\mathrm{c} j}\right) \in$ $\mathbf{R}^{n \times n} \times \mathbf{R}^{n \times p}, j \in\left[0,2^{m}-1\right],\left(\bar{C}_{\mathrm{c}}, \bar{D}_{\mathrm{c}}\right) \in \mathbf{R}^{m \times n} \times \mathbf{R}^{m \times p}$ and $\left(\bar{H}_{C}, \bar{H}_{D}\right) \in \mathbf{R}^{m \times n} \times \mathbf{R}^{m \times p}$ that satisfy (9), (10) and

$$
\left[\begin{array}{ccc}
\frac{1}{\beta} & \bar{H}_{C i} & \bar{H}_{D_{i}} C_{\mathrm{p}} \\
H_{C_{i}^{\mathrm{T}}}^{\mathrm{T}} & R & I \\
C_{\mathrm{p}}^{\mathrm{T}} \bar{H}_{D i}^{\mathrm{T}} & I & S
\end{array}\right] \geq 0, \quad i \in[1, m],
$$

then, the output feedback controller (5) with the controller coefficient matrices given by (11) asymptotically stabilizes the plant (1) at the origin with the ellipsoid $\Omega(X, \beta)$ contained in the domain of attraction.

Proof: We first note that,

$$
\left(x_{\mathrm{c}}, y\right) \in \mathcal{L}\left(H_{C}, H_{D}\right), \quad \text { if }\left(x_{\mathrm{p}}, x_{\mathrm{c}}\right) \in \mathcal{L}\left(H_{D} C_{\mathrm{p}}, H_{C}\right),
$$

where

$$
\begin{gathered}
\mathcal{L}\left(H_{D} C_{\mathrm{p}}, H_{C}\right):=\left\{\left(x_{\mathrm{p}}, x_{\mathrm{c}}\right):\left|H_{D_{i}} C_{\mathrm{p}} x_{p}+H_{C_{i}} x_{\mathrm{c}}\right| \leq 1,\right. \\
i \in[1, m]\} .
\end{gathered}
$$

It thus follows from the proof of Theorem 1 that the closedloop system is asymptotically stable at the origin with $\Omega(X, \beta)$ contained in its domain of attraction if

$$
\Omega(X, \beta) \subset \mathcal{L}\left(H_{D} C_{\mathrm{p}}, H_{C}\right),
$$

which is equivalent to [3]

$$
\beta\left[\begin{array}{ll}
H_{D_{i}} C & H_{C i}
\end{array}\right] X^{-1}\left[\begin{array}{c}
H_{D i}^{\mathrm{T}} C_{\mathrm{p}}^{\mathrm{T}} \\
H_{C i}^{\mathrm{T}}
\end{array}\right] \leq 1, \quad i \in[1, m] .
$$

By Schur complement, the above inequality can be rewritten as

$$
\left[\begin{array}{cc}
\frac{1}{\beta} & {\left[\begin{array}{cc}
H_{D i} C_{\mathrm{p}} & H_{C i}
\end{array}\right]} \\
{\left[\begin{array}{c}
C_{\mathrm{p}}^{\mathrm{T}} H_{D i}^{\mathrm{T}} \\
H_{C i}^{\mathrm{T}}
\end{array}\right]} & X \\
&
\end{array}\right] \geq 0,
$$

which is implied by the condition (17). Indeed, by noting that

$$
\left[\begin{array}{ll}
H_{D} C_{\mathrm{p}} & H_{C}
\end{array}\right] Z_{1}=\left[\begin{array}{ll}
\bar{H}_{C} & \bar{H}_{D} C_{\mathrm{p}}
\end{array}\right],
$$

then multiply $\operatorname{diag}\left\{1, Z_{1}^{\mathrm{T}}\right\}$ from the left-hand side and its transpose from the right-hand side of the inequality (18) reveals that the inequalities (18) and (17) are equivalent.

Theorem 2 provides conditions on the coefficient matrices of the controller under which the ellipsoid $\Omega(X, \beta)$ is inside the domain of attraction. To maximize the cross-section of the ellipsoid in the plant state-space, we introduce a reference ellipsoid $\Omega\left(X_{\mathrm{R}}, 1\right)$ with a given positive definite matrix $X_{\mathrm{R}} \in \mathbf{S}_{+}^{n \times n}$ and maximize $\alpha$ such that

$$
\left.\alpha \Omega\left(X_{\mathrm{R}}, 1\right) \subset \Omega(X, \beta)\right|_{\left(x_{\mathrm{p}}, 0\right)},
$$

which can be converted into an LMI constraint:

$$
\frac{1}{\alpha^{2}} X_{\mathrm{R}}-\frac{S}{\beta} \geq 0 \text {. }
$$

Thus, the determination of the controller coefficient matrices can be formulated and solved as an LMI optimization problem:

$$
\begin{array}{ll} 
& \max \alpha, \\
\text { s.t. } & (19),(9)-(10),(17) .
\end{array}
$$

The output feedback synthesis conditions in Theorems 1, 2 involve only finite number of LMIs. This is due to the specific form of output feedback control law and the use of single quadratic Lyapunov function. It is also possible to consider more general type of output feedback LPV controllers and parameter-dependent Lyapunov functions. However, the resulting synthesis conditions will be infinitedimensional. The solution of such infinite-dimensional LMI optimization problems often require functional space approximation and gridding approach [1], [13].

\section{B. Discrete-Time Systems}

Next, we consider a discrete-time linear system subject to actuator saturation

$$
\left\{\begin{aligned}
x_{\mathrm{p}}(k+1) & =A_{\mathrm{p}} x_{\mathrm{p}}(k)+B_{\mathrm{p}} \sigma(u) \\
y(k) & =C_{\mathrm{p}} x_{\mathrm{p}}(k) .
\end{aligned}\right.
$$

As with continuous-time systems, we will consider the following discrete-time quasi-LPV output feedback controller,

$$
\left\{\begin{aligned}
x_{\mathrm{c}}(k+1)= & \left(\sum_{j=0}^{2^{m}-1} \eta_{j} A_{\mathrm{c} j}\right) x_{\mathrm{c}}(k) \\
& +\left(\sum_{j=0}^{2^{m}-1} \eta_{j} B_{\mathrm{c} j}\right) y(k), \\
u(k)= & C_{\mathrm{c}} x_{\mathrm{c}}(k)+D_{\mathrm{c}} y(k),
\end{aligned}\right.
$$


where $x_{\mathrm{c}} \in \mathbf{R}^{n}$ and the functions $\eta_{j}\left(x_{\mathrm{c}}, y\right)$ 's are defined the same way as in Subsection II-A.

Parallel to the development in Subsection II-A, we have the following results on asymptotic stabilization of the discrete-time plant (21).

Theorem 3: Consider the plant (21). If there exist positive definite matrices $R, S \in \mathbf{S}_{+}^{n \times n}$ and matrices $\bar{A}_{\mathrm{c} j}, \bar{B}_{\mathrm{c} j}, \bar{C}_{\mathrm{c}}, \bar{D}_{\mathrm{c}}$ and $\bar{H}_{C}, \bar{H}_{D}$ of appropriate dimensions such that

$$
\begin{aligned}
& {\left[\begin{array}{cccc}
-R & \star & \star & \star \\
-I & -S & \star & \star \\
A_{\mathrm{p}} R+B_{\mathrm{p}} \bar{K}_{\mathrm{c} j} & A_{\mathrm{p}}+B_{\mathrm{p}} \bar{L}_{\mathrm{c} j} C_{\mathrm{p}} & -R & \star \\
\bar{A}_{\mathrm{c} j} & S A_{\mathrm{p}}+\bar{B}_{\mathrm{c} j} C_{\mathrm{p}} & -I & -S
\end{array}\right]<0,} \\
& {\left[\begin{array}{ccc}
\frac{1}{\beta} & \bar{H}_{C_{i}} & \bar{H}_{D_{i}} C_{\mathrm{p}} \\
\bar{H}_{C i}^{\mathrm{T}} & R & I \\
C_{\mathrm{p}}^{\mathrm{T}} \bar{H}_{D i}^{\mathrm{T}} & I & S
\end{array}\right] \geq 0, \quad i \in[1, m],} \\
& {\left[\begin{array}{cc}
R & I \\
I & S
\end{array}\right]>0,}
\end{aligned}
$$

where $\bar{K}_{\mathrm{c} j}, \bar{L}_{\mathrm{c} j}$ are as defined in Theorem 1, then, the controller (22) with the following coefficient matrices

$$
\begin{gathered}
{\left[\begin{array}{cc}
A_{\mathrm{c} j} & B_{\mathrm{c} j} \\
C_{\mathrm{c}} & D_{\mathrm{c}} \\
H_{C} & H_{D}
\end{array}\right]=} \\
{\left[\begin{array}{ccc}
N & S B_{\mathrm{p}} E_{j} & S B_{\mathrm{p}} E_{j}^{-} \\
0 & I & 0 \\
0 & 0 & I
\end{array}\right]^{-1}\left\{\left[\begin{array}{cc}
\bar{A}_{\mathrm{c} j} & \bar{B}_{\mathrm{c} j} \\
\bar{C}_{\mathrm{c}} & \bar{D}_{\mathrm{c}} \\
\bar{H}_{C} & \bar{H}_{D}
\end{array}\right]\right.} \\
\left.-\left[\begin{array}{cc}
S A_{\mathrm{p}} R & 0 \\
0 & 0 \\
0 & 0
\end{array}\right]\right\}\left[\begin{array}{cc}
M^{\mathrm{T}} & 0 \\
C_{\mathrm{p}} R & I
\end{array}\right]^{-1}
\end{gathered}
$$

asymptotically stabilizes the plant (21) at the origin with the ellipsoid $\Omega(X, \beta)$ contained in the domain of attraction, where $X>0$ is given by

$$
X=\left[\begin{array}{cc}
I & S \\
0 & N^{\mathrm{T}}
\end{array}\right]\left[\begin{array}{cc}
R & I \\
M^{\mathrm{T}} & 0
\end{array}\right]^{-1}
$$

with $M, N \in \mathbf{R}^{n \times n}$ being such that $M N^{\mathrm{T}}=I_{n}-R S$.

Proof: The discrete-time closed-loop system is asymptotically stable if

$$
\begin{aligned}
A_{j}^{\mathrm{T}} X A_{j}-X & <0, \\
\Omega(X, \beta) & \subset \mathcal{L}\left(H_{D} C_{\mathrm{p}}, H_{C}\right), \\
X & >0 .
\end{aligned}
$$

The rest of the proof is similar to that of Theorems 1 and 2.

As with the continuous-time counterpart, the determination of these controller coefficient matrices can be formulated as an LMI optimization problem by maximizing its cross-section area.

$$
\begin{aligned}
\max & \alpha \\
\text { s.t. } & (19),(23)-(25) .
\end{aligned}
$$

\section{AN EXAMPLE}

Let us consider the continuous-time system (1) with

$$
A_{\mathrm{p}}=\left[\begin{array}{cc}
0.6 & -0.8 \\
0.8 & 0.6
\end{array}\right], \quad B_{\mathrm{p}}=\left[\begin{array}{l}
1 \\
3
\end{array}\right], \quad C_{\mathrm{p}}=\left[\begin{array}{ll}
-2 & 2
\end{array}\right] .
$$

A state feedback law for this system was designed in [3]. Solving the optimization problem (20) using a reference circle $x^{\mathrm{T}} x=1$, we obtain a quasi-LPV controller in the form of (5) with $\eta_{1}\left(x_{\mathrm{c}}, y\right)=\lambda_{1}$ and $\eta_{2}\left(x_{\mathrm{c}}, y\right)=1-\lambda_{1}$ and the coefficient matrices given by

$$
\begin{aligned}
A_{\mathrm{c} 0} & =\left[\begin{array}{cc}
-11.977 & 0.033424 \\
0.07319 & -11.768
\end{array}\right], \\
A_{\mathrm{c} 1} & =\left[\begin{array}{cc}
4.7395 & 17.154 \\
-5.0853 & -17.028
\end{array}\right], \\
B_{\mathrm{c} 0} & =\left[\begin{array}{c}
0.1644 \\
-0.0531
\end{array}\right], \quad B_{\mathrm{c} 1}=\left[\begin{array}{c}
7.0990 \times 10^{-3} \\
-4.4275 \times 10^{-3}
\end{array}\right], \\
C_{\mathrm{c}} & =\left[\begin{array}{ll}
10.012 & 10.326
\end{array}\right], \quad D_{\mathrm{c}}=-0.7323 .
\end{aligned}
$$

The auxiliary matrices $H_{C}$ and $H_{D}$ are given by

$$
H_{C}=\left[\begin{array}{ll}
0.3812 & 0.4122
\end{array}\right], \quad H_{D}=-0.6420,
$$

the resulting ellipsoid is $\Omega(X, 1)$ with

$$
X=\left[\begin{array}{ccc}
3.9648 & \multicolumn{2}{c}{-2.1876} \\
-2.1876 & \multicolumn{2}{c}{1.7747} \\
1.0 & -1.2586 \times 10^{-16} \\
0 & \multicolumn{2}{c}{1.0} \\
1.0 & 0 \\
-1.2586 & \times 10^{-16} & 1.0 \\
5.3042 & \times 10^{2} & 1.3558 \times 10^{3} \\
1.3558 & \times 10^{3} & 3.9782 \times 10^{3}
\end{array}\right]
$$

and the maximum $\alpha$ value as 1.11 . In order to reduce controller gain, we have constrained the closed-loop poles in a left-half plane circle $|s+100| \leq 99.5$.

To visualize how our output feedback controller compare with the state feedback law of [3], we plot the cross section of $\Omega(X, \beta)$ and compare it with the two dimensional ellipsoid resulting from the state feedback law (Fig. 1). As can be seen, the output feedback law recovers a significant portion of the stability region resulting from the state feedback law of [3].

Shown in Figs. 2 and 3 are responses of the closed-loop system for large and small initial conditions. In particular, in Fig. 2, the initial condition is $x_{\mathrm{p}}(0)=\left[\begin{array}{ll}0.81 & 2.11\end{array}\right]^{\mathrm{T}}$, for which states converge towards the origin and the control saturates at -1 during initial time.

On the other hand, we choose a small initial condition as $x_{\mathrm{p}}(0)=\left[\begin{array}{ll}-0.05 & 0.2\end{array}\right]^{\mathrm{T}}$ in Fig. 3, for which the control input does not saturate and the state trajectory converges to the origin quickly.

\section{CONCLUSION}

In this paper, we developed a method for the synthesis of output feedback control laws that asymptotically stabilize linear systems subject to actuator saturation. The resulting 


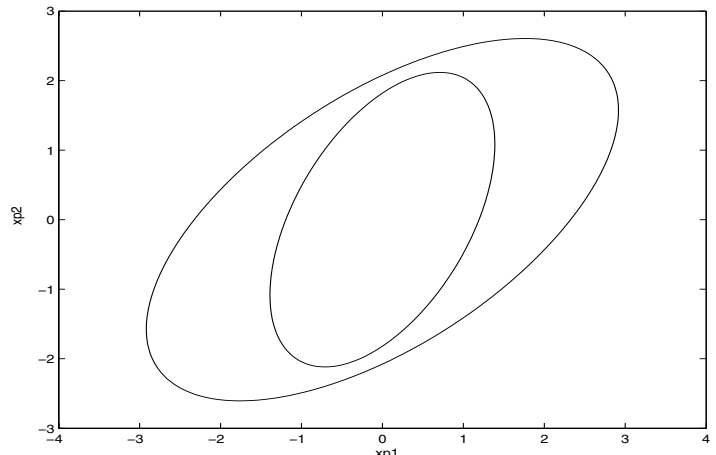

Fig. 1. A cross-section of $\Omega(X, \beta)$ at $x_{\mathrm{c}}=0$.

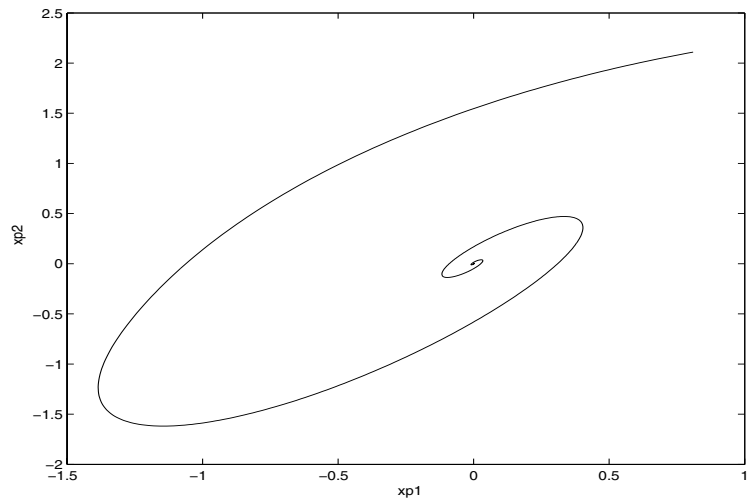

(a) state trajectory

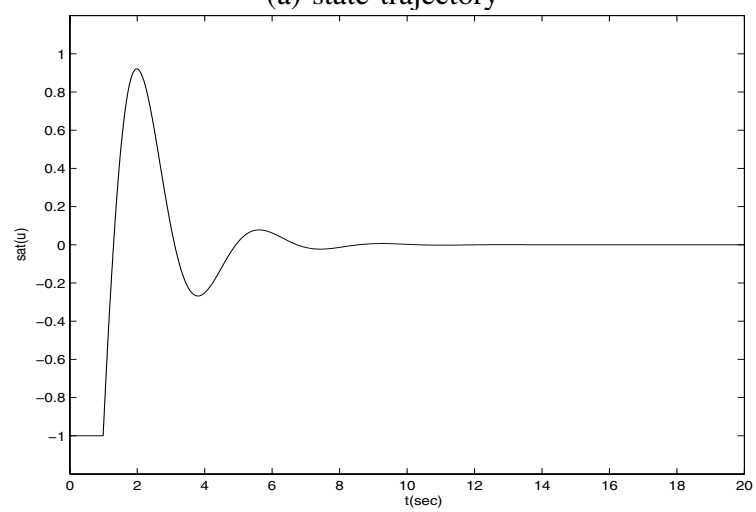

(b) control input

Fig. 2. State trajectory and control input: large initial condition.

output feedback controller is nonlinear in nature, and was parameterized in quasi-LPV form. The determination of the controller coefficient matrices was formulated and solved as an LMI optimization problem, with the objective of enlarging the stability regions. A numerical example is presented to demonstrate the effectiveness of the proposed control design method.

\section{REFERENCES}

[1] G. Becker and A.K. Packard, "Robust performance of linear parametrically varying systems using parametrically-dependent linear feedback," Syst. Contr. Letts., 23:205-215, 1994.

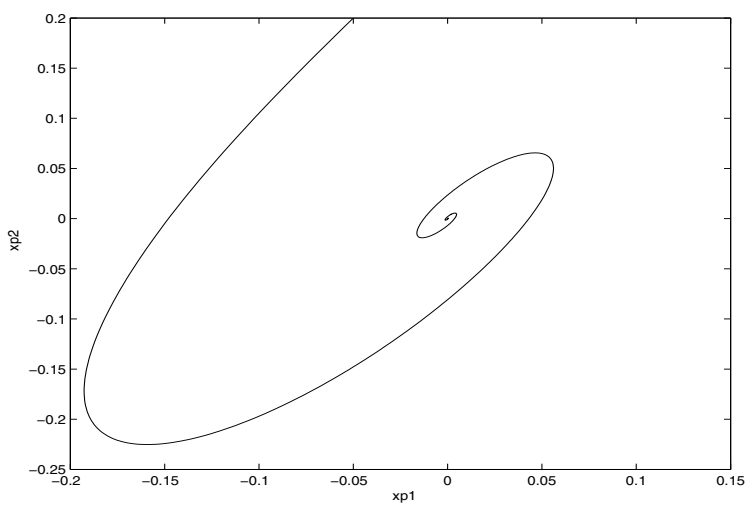

(a) state trajectory

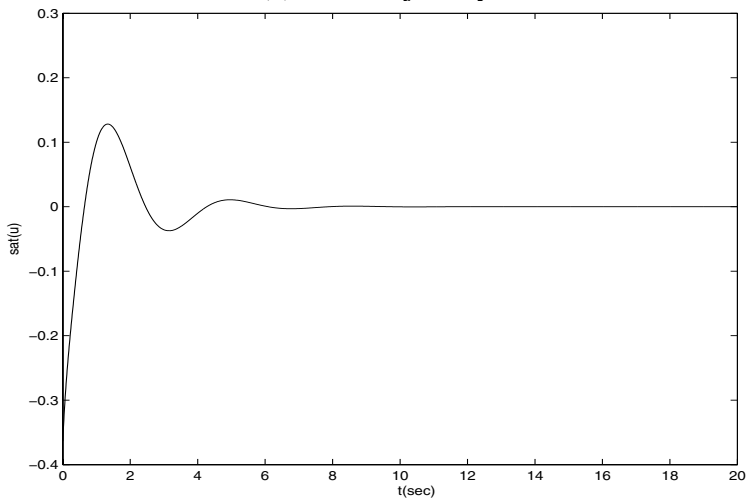

(b) control input

Fig. 3. State trajectory and control input: small initial condition.

[2] H. Hindi and S. Boyd, "Analysis of linear systems with saturating using convex optimization," in Proc. 37th IEEE Conf. Dec. Contr., pp. 903-908, 1998.

[3] T. Hu and Z. Lin, Control Systems with Actuator Saturation: Analysis and Design, Birkhauser, Boston, MA, 2001.

[4] T. Hu and Z. Lin, "On semi-global stabilizability of anti-stable systems by saturated linear feedback," IEEE Trans. Automat. Contr., AC-47(7):1193-1198, 2002.

[5] T. Hu, Z. Lin and B.M. Chen, "An analysis and design method for linear systems subject to actuator saturation and disturbance," Automatica, 38(2):351-359, 2002.

[6] H. Khalil, Nonlinear Systems, Prentice-Hall, Upper Saddle River, NJ, 1996.

[7] Z. Lin and A. Saberi, "Semi-global exponential stabilization of linear systems subject to 'input saturation' via linear feedbacks," Syst. Contr. Letts., 21:225-239, 1993.

[8] Z. Lin and A. Saberi, "Semi-global exponential stabilization of linear discrete-time systems subject to 'input saturation' via linear feedbacks," Syst. Contr. Letts., 24:125-132, 1995.

[9] C. Pittet, S. Tarbouriech and C. Burgat, "Stability regions for linear systems with saturating controls via circle and Popov criteria," in Proc. 36th IEEE Conf. Dec. Contr., pp. 4518-4523, 1997.

[10] R. Suarez, J. Alvarez-Ramirez and J. Solis-Daun, "Linear systems with bounded inputs: global stabilization with eigenvalue placement," Int. J. Robust Non. Contr., 7:835-845, 1997.

[11] H.J. Sussmann, E.D. Sontag and Y. Yang, "A general result on the stabilization of linear systems using bounded controls," IEEE Trans. Automat. Contr., AC-39:2411-2425, 1994.

[12] A.R. Teel, "Global stabilization and restricted tracking for multiple integrators with bounded controls," Syst. Contr. Letts., 18:165-171, 1992.

[13] F. Wu, X.H. Yang, A.K. Packard, and G. Becker, "Induced $\mathcal{L}_{2}$ norm control for LPV systems with bounded parameter variation rates," Int. J. Robust Non. Contr., 6(9/10):983-998, 1996. 